\title{
Prostate cancer with cyst formation detected by whole body positron emission tomography/computed tomography: A case report
}

\author{
HAKUSHI KIM ${ }^{1}$, SUNAO SHOJI ${ }^{1}$, TETSURO TOMONAGA ${ }^{1}$, MASANORI SHIMA ${ }^{1}$, \\ TOSHIRO TERACHI ${ }^{2}$ and TOYOAKI UCHIDA ${ }^{1}$ \\ ${ }^{1}$ Department of Urology, Tokai University Hachioji Hospital, Hachioji, Tokyo 192-0032; \\ ${ }^{2}$ Department of Urology, Tokai University School of Medicine, Isehara, Kanagawa 259-1193, Japan \\ Received February 25, 2014; Accepted July 23, 2014
}

DOI: $10.3892 / \mathrm{ol} .2014 .2510$

\begin{abstract}
The present study reports a case of prostate adenocarcinoma with cyst formation. A 72-year-old male diagnosed with multiple lung metastases at a local clinic was referred to Tokai University Hachioji Hospital (Tokyo, Japan) for detection of a primary lesion. Whole body positron emission tomography/computed tomography showed strong accumulation of $\left[{ }^{18} \mathrm{~F}\right]$-fluoro-deoxy-2-glucose (FDG) in the small pelvis, and pelvic magnetic resonance imaging revealed a $60 \times 40-\mathrm{mm}$ cystic lesion, with an irregular thickened wall, behind the prostate. The serum prostate-specific antigen (PSA) level was elevated to $211.99 \mathrm{ng} / \mathrm{ml}$, therefore, the patient underwent a transperineal prostate needle biopsy, and was diagnosed with prostate adenocarcinoma with cyst formation. Androgen deprivation therapy was administered for 8 months following the diagnosis of prostate cancer; consequently, the cyst reduced in size and the serum PSA level decreased to $0.14 \mathrm{ng} / \mathrm{ml}$. To the best of our knowledge, this is the first report of a malignant prostatic cyst detected by FDG-positron emission tomography/computed tomography.
\end{abstract}

\section{Introduction}

Cysts that form in patients with prostate cancer are a type of acquired cyst (1). In prostate cancer, cysts are either secondary

Correspondence to: Dr Hakushi Kim, Department of Urology, Tokai University Hachioji Hospital, 1838 Ishikawa-machi, Hachioji, Tokyo 192-0032, Japan

E-mail: qblong888@gmail.com

Abbreviations: FDG, $\left[{ }^{18} \mathrm{~F}\right]$-2-fluoro-2-deoxyglucose; PET, positron emission tomography; CT, computed tomography; MRI, magnetic resonance imaging; PSA, prostate-specific antigen; ADT, androgen deprivation therapy

Key words: prostate cancer, cyst formation, positron emission tomography/computed tomography cysts caused by intra-cancerous tissue hemorrhage or central necrosis of the cancer tissue, or primary cysts associated with the cancer (2). The majority of cysts that form in prostate cancer patients are secondary cysts (3). In Japan >100 cases have been reported of prostate cancer with cyst formation $(3,4)$. The majority were symptomatic and detected by ultrasound or computed tomography (CT). In total $>50 \%$ of reported cases presented with metastatic cancer and endocrine therapies were selected for the patient and surgery was only performed for localised disease (5). Nearly all patients were diagnosed with conventional acinar adenocarcinomas from the histology. However, papillary cystadenocarcinomas, embryonal rhabdomyosarcoma and phyllodes tumors are rare (5-7).The present study reports a case of cyst formation in a patient with prostate cancer, secondary to a conventional adenocarcinoma. The patient provided informed consent.

\section{Case report}

A 72-year-old male was diagnosed with multiple lung metastases by chest radiography and CT during a health examination at a local clinic. The patient was referred to Tokai University Hachioji Hospital (Tokyo, Japan) for examination and diagnosis of the primary tumor. Whole body $\left[{ }^{18} \mathrm{~F}\right]$-fluoro-deoxy-2-glucose positron emission tomography (FDG-PET)/CT showed strong accumulation in the pelvis (Fig. 1). Pelvic magnetic resonance imaging (MRI) revealed a 60x40-mm cystic lesion, with an irregular thickened wall, behind the left lobe of the prostate (Fig. 1); this finding was consistent with the FDG accumulation observed on PET/CT. A transperineal needle biopsy was performed once the serum prostate-specific antigen (PSA) level was found to be elevated to $211.99 \mathrm{ng} / \mathrm{ml}$ (normal range, $4.0 \mathrm{ng} / \mathrm{ml}$ ). Histological examination of the needle biopsy specimens of the cystic wall and prostate gland revealed moderately-differentiated adenocarcinoma (Gleason score 4+3) (8). The contents of the cyst were bloody. The cytological findings revealed no malignancy, but the PSA level of the cystic contents was $45,130 \mathrm{ng} / \mathrm{ml}$. Whole body $\mathrm{CT}$ and bone scans revealed no metastasis other than that in the lung, and the patient was diagnosed with prostate cancer with multiple lung metastases. Following 8 months of 

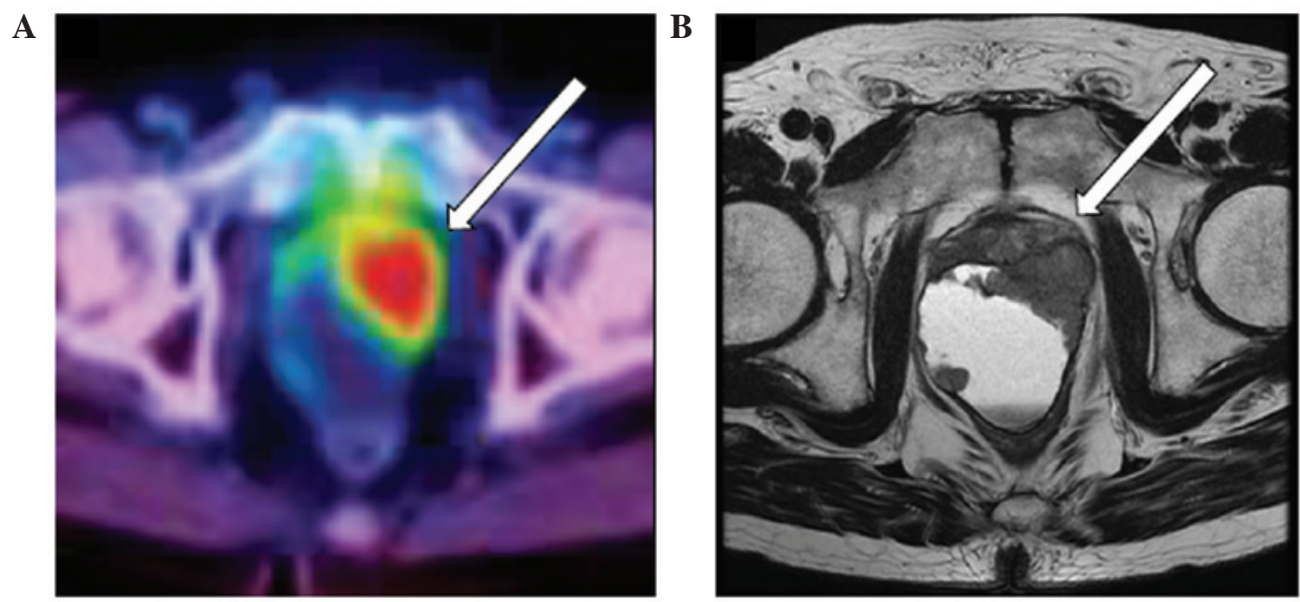

Figure 1. (A) $\left[{ }^{18} \mathrm{~F}\right]$-fluoro-deoxy-2-glucose positron emission tomography/computed tomography showing strong accumulation in the pelvis (arrow), and (B) pelvic magnetic resonance imaging showing a cystic lesion (arrow) behind the prostate gland. The wall of the cyst is partially thickened, and the surface of the thickened wall is irregular.

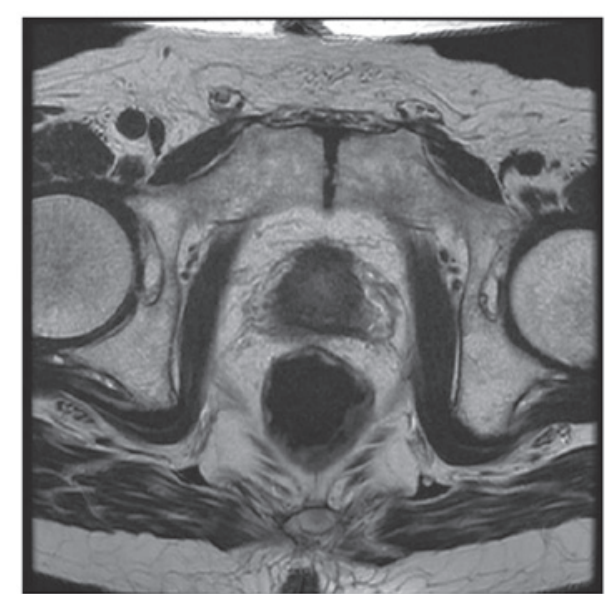

Figure 2. Pelvic magnetic resonance imaging following 8 months of androgen deprivation therapy showing shrinkage of the cyst.

androgen deprivation therapy (ADT), the cyst shrank (Fig. 2) and the serum PSA level decreased to $0.14 \mathrm{ng} / \mathrm{ml}$. At the 24-month post-ADT follow-up examination, the PSA level was $0.19 \mathrm{ng} / \mathrm{ml}$ and the cyst continued to shrink.

\section{Discussion}

In prostate cancer, cysts are considered either secondary cysts caused by intra-cancerous tissue hemorrhage or necrosis of the cancer tissue, or primary cysts associated with cancer (2). In a recent Japanese study of 96 patients with prostate cancer with cyst formation, the majority were symptomatic (dysuria was reported in $56.2 \%$ of cases and hematuria in $20.8 \%$ of cases), more than half presented with metastatic cancer $(54.1 \%)$ and almost all were diagnosed with secondary cyst formation $(82.1 \%)$ (3). In the present case, although a cyst with a diameter of $\sim 6 \mathrm{~cm}$ was detected in the small pelvis, the patient was asymptomatic.

It is difficult to predict tissue type without histological examination by MRI or PET/CT. Although only the pathological data acquired by prostate biopsy was obtained, the cystic fluid was bloody with a high PSA level. Based on these results, we speculate that the cyst was a secondary cyst that formed in the prostate cancer patient.

The various histological types of malignant lesions with gross cystic sections in the prostate have been previously reported in the literature, and include conventional acinar adenocarcinomas, papillary cystadenomas (4), embryonal rhabdomyosarcomas (6) and phyllodes tumors (7). Ideally, the histological type of prostate cancer should be determined prior to the start of treatment. The present patient did not exhibit the typical clinical manifestation of conventional acinar adenocarcinoma, but the pathological examination indicated acinar prostate adenocarcinoma. Therefore, the standard therapy for metastatic prostate adenocarcinoma was chosen, which was extremely effective.

FDG-PET/CT is of limited value for detecting prostate cancer, as only $\sim 1 \%$ of prostate cancer lesions are FDG-avid (9) and benign conditions of the prostate can also show increased FDG uptake (10). However, there is evidence in the literature that FDG-PET/CT sensitivity and a positive predictive value to detect prostate cancer is increased up to 80 and $87 \%$, respectively, in tumors classified with a Gleason score of $\geq 7$ (11). In the present case, the thickened wall of the cyst that continued to the left lobe of the prostate showed a high accumulation of tracer, which aided in the decision with regard to which part of the body to examine to identify the primary lesion of the lung metastases.

\section{References}

1. Emmett JL and Braasch WF: Cysts of the prostate gland. J Urol 36: 236-249, 1936.

2. Kojima K, Uehara H, Naruo S, Kanayama H and Kagawa S: Papillary cystadenocarcinoma of the prostate. Int J Urol 3: 511-513, 1996.

3. Itami Y, Nagai Y, Kobayashi Y, Shimizu N, Yamamoto Y, Minami T, Hayashi T, Nozawa M, Yoshimura K, Ishii T and Uemura $\mathrm{H}$ : A case of prostatic cancer with a low PSA level accompanied with cystic formation requiring differentiation from adenocarcinoma of the seminal vesicle. Hinyokika Kiyo 58: 349-353, 2012 (In Japanese).

4. Naoe M, Ogawa Y, Fuji K, Fukagai T, Inoue K and Yoshida H: Papillary cystadenocarcinoma of the prostate. Int J Urol 11: 1036-1038, 2004. 
5. Kim SC1, Fujimoto K, Matsumoto Y, et al: A case of prostate cancer with cyst formation. Hinyokika Kiyo 47: 653-656, 2001 (In Japanese).

6. Niimi K, Hashimoto Y, Kurokawa S, Okada A, Tozawa K and Kohri K: Embryonal rhabdomyosarcoma of the prostate. Int J Clin Oncol 15: 93-96, 2010.

7. Chung HC, Lee HS, Kim TI, Kim DI, Park KH and Song JM: A large cystic phyllodes tumor of the prostate. Yonsei Med J 50: 174-176, 2009.

8. Epstein JI, Allsbrook WC Jr, Amin MB, et al: The 2005 International Society of Urological Pathology (ISUP) Consensus Conference on Gleason Grading of prostatic carcinoma. Am J Surg Pathol 29: 1228-1242, 2005.
9. Hinev A, Chaushev B and Klisarova A: FDG PET/CT in prostate cancer: A valuable method to detect the primary and metastatic tumor sites and to monitor cancer response to hormonal therapy. Nephrourol Mon 4: 644-645, 2012.

10. Han EJ, JH O, Choi WH, Yoo IR and Chung SK: Significance of incidental focal uptake in prostate on 18-fluoro-2-deoxyglucose positron emission tomography CT images. Br J Radiol 83: 915-920, 2010

11. Minamimoto R, Uemura H, Sano F, Terao H, Nagashima Y, Yamanaka S, Shizukuishi K, Tateishi U, Kubota Y and Inoue T: The potential of FDG-PET/CT for detecting prostate cancer in patients with an elevated serum PSA level. Ann Nucl Med 25: 21-27, 2011. 